\title{
How useful are ARFI elastography cut-off values proposed by meta- analysis for predicting the significant fibrosis and compensated liver cirrhosis?
}

\author{
Simona Bota, Ioan Sporea, Roxana Şirli, Alina Popescu, Oana Gradinaru-Tașcău
}

Department of Gastroenterology and Hepatology, "Victor Babeș” University of Medicine and Pharmacy Timișoara, Romania

\begin{abstract}
Aim: To evaluate how often do we "miss" chronic hepatitis $\mathrm{C}$ patients with at least significant fibrosis $(\mathrm{F} \geq 2)$ and those with compensated cirrhosis, by using Acoustic Radiation Force Impulse (ARFI) elastography cut-off values proposed by meta-analysis. Material and methods: Our study included 132 patients with chronic hepatitis C, evaluated by means of ARFI and liver biopsy (LB), in the same session. Reliable measurements were defined as: median value of 10 liver stiffness (LS) measurements with a success rate $\geq 60 \%$ and an interquartile range interval $<30 \%$. For predicting $F \geq 2$ and $F=4$ we used the LS cut-offs proposed in the last published meta-analysis: $1.35 \mathrm{~m} / \mathrm{s}$ and $1.87 \mathrm{~m} / \mathrm{s}$, respectively. Results: Reliable LS measurements by means of ARFI were obtained in 117 patients (87.9\%). In our study, 58 patients (49.6\%) had LS values $<1.35 \mathrm{~m} / \mathrm{s}$; from these $75.8 \%$ had $\mathrm{F} \geq 2$ in LB. From the 59 patients $(50.4 \%)$ with LS values $\geq 1.35 \mathrm{~m} / \mathrm{s}$, only $6.8 \%$ had F0 or F1 in LB. Also, in our study, 88 patients $(75.3 \%)$ had LS values $<1.87 \mathrm{~m} / \mathrm{s}$; from these only $2.2 \%$ had F4 in LB. From the 29 patients $(24.7 \%)$ with LS values $\geq 1.87 \mathrm{~m} / \mathrm{s}, 41.3 \%$ had F4 in LB. Both for prediction of at least significant fibrosis and liver cirrhosis, higher aminotransferases levels were associated with wrongly classified patients, in univariate and multivariate analysis. Conclusions: ARFI elastography had a very good positive predictive value (93.2\%) for predicting the presence of significant fibrosis and excellent negative predictive value (97.8\%) for excluding the presence of compensated liver cirrhosis.
\end{abstract}

Keywords: liver fibrosis, liver stiffness, chronic hepatitis C, liver cirrhosis, ARFI elastography

\section{Introduction}

Chronic infection with hepatitis $\mathrm{C}$ virus is still an important public health concern and evaluation of liver fibrosis is essential for prognosis and treatment decision in these patients. Liver fibrosis assessment can be performed using invasive (liver biopsy-LB) or non-invasive methods, which include elastography $[1,2]$ or serological tests $[3,4]$.

Acoustic Radiation Force Impulse (ARFI) elastography is a "point" shear wave elastographic method. The

Received 23.03.2015 Accepted 16.04.2015

Med Ultrason

2015, Vol. 17, No 2, 200-205

Corresponding author: Simona Bota,

2, Intrarea Martir Angela Sava street

300742, Timisoara, Romania

Phone: +40-721-656147, Fax: +40-256-488003

E-mail: bota_simona1982@yahoo.com applied force is dynamic and ultrasound induced. Tissue displacement is produced using an acoustic radiation force impulse that induces a smaller strain in hard tissues than in soft ones. The shear waves speed is measured by the ultrasound device and expressed in meters/second $(\mathrm{m} / \mathrm{s})[1,5]$.

In the last years several studies and meta-analyses [6-8] regarding the usefulness of this technique for non-invasive assessment of liver fibrosis were published, but no significant information regarding the usefulness of meta-analyses cut-offs for predicting different stages of liver fibrosis in daily clinical practice are available.

The aim of our study was to evaluate how often do we "miss" chronic hepatitis C patients with at least significant fibrosis $(\mathrm{F} \geq 2)$ and those with compensated cirrhosis $(\mathrm{F}=4)$ by using ARFI cut-off values proposed by a metaanalysis. 


\section{Material and methods}

\section{Patients}

Our retrospective study included 132 patients with chronic hepatitis $C$ evaluated in our Department in the same session by means of ARFI elastography and LB(and most of them also by Transient Elastography), between October 2009-April 2013. The diagnosis of chronic infection with hepatitis $\mathrm{C}$ virus was established as follows: positive serum anti-HCV antibodies for at least 6 months and detectable hepatitis $\mathrm{C}$ virus RNA in serum, by real-time polymerase chain reaction (PCR ARN-HCV). None of the patients had coinfection with hepatitis B virus or human immunodeficiency virus. None of the patients had liver focal liver lesions or ascites on abdominal ultrasound examination. Our study was approved by the local Ethics Committee and was in accordance with the Helsinki Declaration of 1975.

\section{ARFI elastography}

ARFI was performed in all patients, in fasting condition, with a Siemens Acuson S2000 ${ }^{\mathrm{TM}}$ ultrasound system using Virtual Touch Tissue Quantification application (Siemens AG, Erlangen, Germany) with a 4CI transducer. Scanning was performed between the ribs with the patient in supine position, in the right liver lobe (segment V/VIII). The "box" with a predefined size of $5 \mathrm{~mm}$ width and $10 \mathrm{~mm}$ length was positioned by the operator in a region free of vessels, $1-2 \mathrm{~cm}$ under the liver capsule and then a minimal scanning pressure was applied, while the patients were asked to stop breathing for a moment, in order to minimize breathing motions. In every patient we aimed for 10 valid LS measurements performed in the same place in the right liver lobe and a median value was calculated, the result being measured in $\mathrm{m} / \mathrm{s}$. If the measurement was not valid, "X.Xx" was displayed on the screen. Reliable LS measurements were defined as median value of 10 valid measurements with an interquartile range interval (IQR) $<30 \%$ and a success rate $\geq 60 \%$.

\section{Transient Elastography}

Transient Elastography was performed using a FibroScan ${ }^{\circledR}$ device (EchoSens, Paris, France) (standard Mprobe) and was available in $123 / 132$ patients (93.1\%). In each patient we aimed for 10 valid TE measurements using the standard M-probe. The LS measurements were performed under fasting conditions, in supine position, by intercostal approach, with the right arm in maximum abduction; then a median value was calculated and the results were expressed in kiloPascals $(\mathrm{kPa})$. Reliable measurements were defined as: median value of 10 valid LS measurements with IQR $<30 \%$ and $\mathrm{SR} \geq 60 \%$.

\section{Liver biopsy}

Liver biopsy was performed echo-assisted using Menghini type modified needles, 1.4 and $1.6 \mathrm{~mm}$ in diameter. All liver specimens were at least $2 \mathrm{~cm}$ long (a quality parameter that we use in daily practice in our department). The biopsy fragment's length was evaluated by the physician who performed the procedure.

The LBs were assessed according to the Metavir score, by a senior pathologist, blinded to the results of ARFI measurements. Fibrosis was staged on a 0-4 scale: F0 - no fibrosis; F1 - portal fibrosis without septa; F2 - portal fibrosis and few septa extending into lobules; F3 - numerous septa extending to adjacent portal tracts or terminal hepatic venules and F4 - cirrhosis. Activity was graded on a 0-3 scale: A0 - no activity, A1 - mild activity, A2 - moderate activity and A3 - severe activity. When steatosis was present, it was assessed according to the Hepburn classification into 5 categories, based on the percentage of hepatocytes affected by steatosis on biopsy: $<2 \%, 2-10 \%, 10-30 \%, 30-60 \%$, and $>60 \%$.

\section{Statistical analysis}

The statistical analysis was performed using the MedCalc Software, version 12.7.0 (MedCalc program, Belgium). The distribution of numerical variables was first tested by the Kolmogrov-Smirnov test, numerical variables being presented as mean \pm standard deviation or median with range interval according to their normal or non-normal distribution. Differences between numerical variables were analyzed by parametric tests (t-test) in cases of variables with normal distribution, otherwise nonparametric tests (Mann-Whitney test) where applied. Chi-square $\left(\mathrm{X}^{2}\right)$ test was used for comparing proportions expressed as percentages ("n" designates the total number of patients included in a particular subgroup). 95\% confidence intervals were calculated for each predictive test and a $p$-value less than 0.05 was regarded as significant for each statistic test.

The performance of ARFI elastography for predicting the presence of at least significant fibrosis (F2) and liver cirrhosis (F4) was assessed using the cut-offs recently published in a meta-analysis [8]: $1.35 \mathrm{~m} / \mathrm{s}$ and $1.87 \mathrm{~m} / \mathrm{s}$, respectively. We analyzed in univariate analysis if the following factors were associated with wrongly classified patients: age, gender, body mass index (BMI), length of LB specimen, number of portal tracts, high aminotransferases level and steatosis $\geq 30 \%$. In the second step, the independent discriminative values of variables reaching a univariate statistic significance $(p<0.05)$ where assessed by backward stepwise binary multiple regression analysis.

For TE, we used the cut-offs values for chronic hepatitis $\mathrm{C}$ patients proposed by the most recently published meta-analysis [9]: $7.6 \mathrm{kPa}$ for significant fibrosis (F2) and $15.3 \mathrm{kPa}$ for liver cirrhosis (F4 Metavir). 
Table I. Main patients' characteristics.

\begin{tabular}{|c|c|}
\hline Parameter & \\
\hline Age (years) & $53(21-65)$ \\
\hline Gender: - female & $n=87(65.9 \%)$ \\
\hline- male & $\mathrm{n}=45(34.1 \%)$ \\
\hline BMI $\left(\mathrm{kg} / \mathrm{m}^{2}\right)$ & $26.1 \pm 4.3$ \\
\hline Distribution of fibrosis on LB: $-\mathrm{F} 0$ & $\mathrm{n}=7(5.3 \%)$ \\
\hline$-\mathrm{F} 1$ & $\mathrm{n}=12(9.1 \%)$ \\
\hline$-\mathrm{F} 2$ & $\mathrm{n}=58(43.9 \%)$ \\
\hline$-\mathrm{F} 3$ & $n=39(29.6 \%)$ \\
\hline$-\mathrm{F} 4$ & $\mathrm{n}=16(12.1 \%)$ \\
\hline Distribution of activity on LB: $-\mathrm{A} 0$ & $\mathrm{n}=0(0 \%)$ \\
\hline$-\mathrm{A} 1$ & $\mathrm{n}=8(6.1 \%)$ \\
\hline$-\mathrm{A} 2$ & $\mathrm{n}=68(51.5 \%)$ \\
\hline$-\mathrm{A} 3$ & $\mathrm{n}=56(42.4 \%)$ \\
\hline Distribution of steatosis on LB: & $n=45(34.1 \%)$ \\
\hline- absent & $n=30(22.7 \%)$ \\
\hline$-<2 \%$ (Hepburn I) & $n=30(22.7 \%)$ \\
\hline$-2-10 \%$ (Hepburn II) & $\mathrm{n}=12(9.1 \%)$ \\
\hline - 10-30\% (Hepburn III) & $n=14(10.6 \%)$ \\
\hline$-30-60 \%$ (Hepburn IV) & $\mathrm{n}=1(0.8 \%)$ \\
\hline$->60 \%($ Hepburn V) & \\
\hline ALT (U/L) x upper limit of normal (ULN) & $1.5(0.5-8)$ \\
\hline Length of LB specimen $(\mathrm{cm})$ & $3.5(2-6)$ \\
\hline Number of portal tracts & $26.9 \pm 10.1$ \\
\hline
\end{tabular}

Numerical variables with normal distribution are presented as mean value \pm standard deviation, while variables with non-normal distribution are presented as median values and range intervals; BMI - body mass index; ALT - alanine aminotransferase

\section{Results}

The main characteristics of the 132 chronic hepatitis $\mathrm{C}$ patients included in this study are presented in table I.
Reliable LS measurements by means of ARFI elastography were obtained in $117 / 132$ patients $(87.9 \%)$, patients included in the final analysis.

In our cohort of patients, $58(49.6 \%)$ had LS values $<$ $1.35 \mathrm{~m} / \mathrm{s}$; from these $75.8 \%$ had $\mathrm{F} \geq 2$ in LB. From the 59 patients $(50.4 \%)$ with $\mathrm{LS}$ values $\geq 1.35 \mathrm{~m} / \mathrm{s}$, only $6.8 \%$ had $\mathrm{F} 0$ or F1 in LB, meaning $93.2 \%$ positive predictive value (PPV) for predicting the presence of at least significant fibrosis.

In univariate analysis higher aminotransferases level, female gender and shorter length of liver biopsy specimen were associated with wrongly classified patients for predicting the presence of at least significant fibrosis (Table II).

All the factors associated in univariate analysis with wrongly classified patients for $\mathrm{F} \geq 2$ reached the statistical significant also in multivariate analysis (Table III).

From the 44 patients with at least significant fibrosis on LB and LS values by ARFI elastography $<1.35$ $\mathrm{m} / \mathrm{s}$, TE was available in 41/44 cases (93.2). Reliable LS measurements were obtained in $38 / 41$ cases and only 9/38 (23.6\%) patients had LS values $\geq 7.6 \mathrm{kPa}$.

In our cohort of patients, 88 patients $(75.3 \%)$ had LS values $<1.87 \mathrm{~m} / \mathrm{s}$; from these only $2.2 \%$ had F4 in LB, meaning $97.8 \%$ negative predictive value (NPV) for excluding the presence of liver cirrhosis. From the 29 patients $(24.7 \%)$ with LS values $\geq 1.87 \mathrm{~m} / \mathrm{s}, 41.3 \%$ had cirrhosis in LB.

Higher aminotransferases levels and shorter length of liver biopsy specimen were associated in univariate analysis with wrongly classified patients for predicting

Table II. Factors associated with wrongly classified patients for predicting the presence of at least significant fibrosis (univariate analysis)

\begin{tabular}{llll}
\hline Parameter & Correctly classified & Wrong classified & p value \\
\hline Age (years) & $52(21-65)$ & $53(21-65)$ & 0.37 \\
Female gender & $\mathrm{n}=35(50.7 \%)$ & $\mathrm{n}=41(85.4 \%)$ & $\mathbf{0 . 0 0 0 2}$ \\
BMI (kg/m2) & $25.8 \pm 4.1$ & $25.7 \pm 4.3$ & 0.58 \\
Length of LB specimen (cm) & $4(2-6)$ & $3(2-5)$ & $\mathbf{0 . 0 1}$ \\
Portal tracts & $26(15-55)$ & $23(4-46)$ & 0.09 \\
ALT (U/L) & $1.2 \times$ ULN $(0.5-5 \times$ ULN) & $2 \times$ ULN $(0.8-8 \times$ ULN) & $\mathbf{0 . 0 1}$ \\
Steatosis $\geq 30 \%$ (Hepburn IV and V) & $\mathrm{n}=7(10.1 \%)$ & $\mathrm{n}=6(12.5 \%)$ & 0.91 \\
\hline
\end{tabular}

BMI - body mass index; LB - liver biopsy; ALT - alanine aminotransferase

Table III. Factors associated with wrongly classified patients for predicting the presence of at least significant fibrosis (multivariate analysis)

\begin{tabular}{llll}
\hline Parameter & Coefficient & Standard error & p value \\
\hline ALT (U/L) & 0.114 & 0.029 & $\mathbf{0 . 0 0 0 2}$ \\
Length of LB specimen (cm) & 0.150 & 0.050 & $\mathbf{0 . 0 0 3}$ \\
Female vs. male gender & -0.427 & 0.089 & $<\mathbf{0 . 0 0 0 1}$ \\
\hline
\end{tabular}

ALT - alanine aminotransferase; LB - liver biopsy 
Table IV. Factors associated with wrongly classified patients for predicting the presence of compensated liver cirrhosis (univariate analysis).

\begin{tabular}{llll}
\hline Parameter & Correctly classified & Wrong classified & p value \\
\hline Age (years) & $53(21-65)$ & $54(37-64)$ & 0.32 \\
Female gender & $\mathrm{n}=61(61.6 \%)$ & $\mathrm{n}=15(83.3 \%)$ & 0.13 \\
BMI $(\mathrm{kg} / \mathrm{m} 2)$ & $25.5 \pm 4.3$ & $27.1 \pm 2.8$ & 0.12 \\
Length of LB specimen $(\mathrm{cm})$ & $4(3-6)$ & $3.5(2-5)$ & $\mathbf{0 . 0 3}$ \\
Portal tracts & $27(15-55)$ & $25(4-47)$ & 0.06 \\
ALT (U/L) & $1.3 \times$ ULN $(0.5-8 \times$ ULN) & $2.4 \times$ ULN $(0.7-6 \times$ ULN) & $\mathbf{0 . 0 0 4}$ \\
Steatosis $\geq 30 \%$ (Hepburn IV & $\mathrm{n}=10(10.1 \%)$ & $\mathrm{n}=3(16.6 \%)$ & 0.68 \\
and V) & & & \\
\hline
\end{tabular}

BMI- body mass index; ALT- alanine aminotransferase; LB- liver biopsy

Table V. Factors associated with wrongly classified patients for predicting the presence of compensated liver cirrhosis (multivariate analysis).

\begin{tabular}{llll}
\hline Parameter & Coefficient & Standard error & p value \\
\hline ALT (U/L) & 0.058 & 0.028 & $\mathbf{0 . 0 4}$ \\
Length of LB specimen $(\mathrm{cm})$ & 0.098 & 0.050 & 0.055 \\
Portal tracts & 0.0015 & 0.004 & 0.74 \\
\hline
\end{tabular}

ALT- alanine aminotransferase; LB- liver biopsy

the presence of compensated liver cirrhosis (Table IV), but in multivariate analysis only aminotransferases levels reached statistical significance (Table V).

From the 17 patients with LS values by ARFI elastography $\geq 1.87 \mathrm{~m} / \mathrm{s}$, but without cirrhosis at LB, TE was available in all the cases. Reliable LS measurements were obtained in 14/17 cases and in all cases the LS values by TE were lower than $15.3 \mathrm{kPa}$.

\section{Discussions}

The "gold-standard" method for liver fibrosis evaluation is still considered to be $\mathrm{LB}$, but it is an invasive method [10] which evaluates only $1 / 50000$ of the total volume of the liver [11]. Also, the LB specimen must meet certain quality criteria $[12,13]$, which is not always possible in daily clinical practice [14]. Another issue of LB is the pathologists' agreement rate for liver fibrosis evaluation, reported to be only moderate in most published studies $[11,15]$. Starting from these weak points of LB, non-invasive methods for liver fibrosis assessment were developed, the most used in daily practice being TE and serological tests. The usefulness of Transient Elastography was recognized by the European Association for the Study of the Liver (EASL) by including it as an assessment tool in the guidelines for liver fibrosis evaluation in chronic hepatitis $\mathrm{B}$ and $\mathrm{C}$ patients $[16,17]$.

In the last 3-4 years several studies and three metaanalyses [6-8] demonstrated the good performance of ARFI elastography for the non-invasive evaluation of liver fibrosis, and especially for predicting severe fibrosis and cirrhosis. Also, the meta-analysis published by Bota et al [7] showed that ARFI elastography and TE have similar value for predicting significant fibrosis and cirrhosis.

The aim of our study was to analyze how often do we "miss" chronic hepatitis C patients with at least significant fibrosis and those with compensated cirrhosis, by using ARFI cut-off values proposed by a meta-analysis which included almost 4000 patients [8].

Our results showed that $93.2 \%$ of patients with LS values assessed by ARFI elastography higher than 1.35 $\mathrm{m} / \mathrm{s}$ had at least significant fibrosis on LB, so a very good PPV. However, in the cohort of patients with LS values lower than this cut-off, $75.8 \%$ had $\mathrm{F} \geq 2$ on LB. In these patients neither TE was useful for predicting the presence of at least significant fibrosis, since only $23.6 \%$ of them had LS values higher than the cut-off proposed for chronic hepatitis $\mathrm{C}$ patients in the meta-analysis published by Tsochatzis et al (7.6 kPa) [9]. Considering the fact that due to limited economic resources, in some countries, not all chronic hepatitis $\mathrm{C}$ patients can receive antiviral treatment, ARFI elastography can be a useful method to select those with at least significant fibrosis who need to be treated as quickly as possible. Thus, if LS values by ARFI are at least $1.35 \mathrm{~m} / \mathrm{s}$, the patients can receive antiviral treatment, but if the ARFI elastography values are lower than this cut-off, a LB should be performed.

Another argument to use ARFI elastography in chronic hepatitis C patients is that LS values seem to be asso- 
ciated with the treatment outcome, namely significantly decreasing in patients who achieve sustained virologic response [18].

The diagnosis of compensated liver cirrhosis is very important (and not always easy to establish) for patients with chronic liver diseases, because these subjects need special care, such as screening for hepatocellular carcinoma or for esophageal varices. Our study showed an excellent NPV (97.8\%) for excluding the presence of cirrhosis when LS values assessed by ARFI elastography were lower than $1.87 \mathrm{~m} / \mathrm{s}$. Unfortunately the PPV was not so god, only $41.3 \%$ of patients with LS values higher than $1.87 \mathrm{~m} / \mathrm{s}$ having cirrhosis on LB. We propose that patients with ARFI elastography values suggesting liver cirrhosis to be evaluated also by TE, knowing that in our study all patients without histological cirrhosis had LS values by TE lower than $15.3 \mathrm{kPa}$.

For both prediction of at least significant fibrosis and compensated liver cirrhosis, higher aminotransferases levels were associated with wrongly classified patients, both in univariate and multivariate analysis. Our data is another argument in favor of already published studies, which showed that elevated aminotransferases level are a confounding factor for non-invasive LS evaluation by means of ARFI elastography $[19,20]$, so LS values should be interpreted with caution in this situation. It should be specified that elevated aminotransferases are a confounding factor for TE also [21,22].

According to our data, both in univariate and multivariate analysis, female gender and shorter length of liver biopsy specimen were associated with wrongly classified patients for predicting the presence of at least significant fibrosis. We don't have a plausible explanation for female gender association with wrongly classified subjects. Regarding the length of LB specimen, our study shows once again its' importance for an accurate assessment of the liver disease.

In conclusion, ARFI elastography had a very good PPV (93.2\%) for predicting the presence of significant fibrosis and excellent NPV (97.8\%) for excluding the presence of compensated liver cirrhosis. For both prediction of at least significant fibrosis and liver cirrhosis, higher aminotransferases levels were associated with wrongly classified patients.

\section{Acknowledgments}

For the young researchers grant „, Utilitatea metodelor elastografice de tip shear-waves în evaluarea pacienților cu ciroză hepatică” awarded by the „Victor Babeș” University of Medicine and Pharmacy, Timișoara, Romania.

Conflict of interest: none

\section{References}

1. Bamber J, Cosgrove D, Dietrich CF, et al. EFSUMB guidelines and recommendations on the clinical use of ultrasound elastography. Part 1: Basic principles and technology. Ultraschall Med 2013; 34: 169-184.

2. Cosgrove D, Piscaglia F, Bamber J, et al. EFSUMB guidelines and recommendations on the clinical use of ultrasound elastography. Part 2: Clinical applications. Ultraschall Med 2013; 34: 238-253.

3. Poynard T, Imbert-Bismut F, Ratziu V, et al; GERMED cyt04 group. Biochemical markers of liver fibrosis in patients infected by hepatitis $\mathrm{C}$ virus: longitudinal validation in a randomized trial. J Viral Hepat 2002; 9: 128-133.

4. Guéchot J, Trocmé C, Renversez JC, Sturm N, Zarski JP; ANRS HC EP 23 Fibrostar Study Group. Independent validation of the Enhanced Liver Fibrosis (ELF) score in the ANRS HC EP 23 Fibrostar cohort of patients with chronic hepatitis C. Clin Chem Lab Med 2012; 50: 693-699.

5. Palmeri ML, Wang MH, Dahl JJ, Frinkley KD, Nightingale KR. Quantifying hepatic shear modulus in vivo using acoustic radiation force. Ultrasound Med Biol 2008; 34: 546-558

6. Friedrich-Rust M, Nierhoff J, Lupsor M, et al. Performance of Acoustic Radiation Force Impulse imaging for the staging of liver fibrosis: a pooled meta-analysis. J Viral Hepat 2012; 19: e212-e219.

7. Bota S, Herkner H, Sporea I, et al. Meta-analysis: ARFI elastography versus transient elastography for the evaluation of liver fibrosis. Liver Int 2013; 33: 1138-1147.

8. Nierhoff J, Chávez Ortiz AA, Herrmann E, Zeuzem S, Friedrich-Rust M. The efficiency of acoustic radiation force impulse imaging for the staging of liver fibrosis: a metaanalysis. Eur Radiol 2013; 23: 3040-3053.

9. Tsochatzis EA, Gurusamy KS, Ntaoula S, Cholongitas E, Davidson BR, Burroughs AK. Elastography for the diagnosis of severity of fibrosis in chronic liver disease: a metaanalysis of diagnostic accuracy. J Hepatol 2011; 54: 650-659.

10. Rockey DC, Caldwell SH, Goodman ZD, Nelson RC, Smith AD; American Association for the Study of Liver Diseases. Liver biopsy. Hepatology 2009; 49: 1017-1044.

11. Regev A, Berho M, Jeffers LJ, et al. Sampling error and intraobserver variation in liver biopsy in patients with chronic HCV infection. Am J Gastroenterol 2002; 97: 2614-2618.

12. Friedman LS. Controversies in liver biopsy: who, where, when, how, why? Curr Gastroenterol Rep 2004; 6: 30-36.

13. Guido M, Rugge M. Liver biopsy sampling in chronic viral hepatitis. Semin Liver Dis 2004; 24: 89-97.

14. Cholongitas E, Senzolo M, Standish R, et al. A systematic review of the quality of liver biopsy specimens. Am J Clin Pathol 2006; 125: 710-721.

15. Rousselet MC, Michalak S, Dupré F, et al; Hepatitis Network 49. Sources of variability in histological scoring of chronic viral hepatitis. Hepatology 2005; 41: 257-264.

16. European Association for the Study of the Liver. EASL Clinical Practice Guidelines: Management of hepatitis $\mathrm{C}$ virus infection. J Hepatol 2014; 60: 392-420. 
17. European Association For The Study Of The Liver. EASL clinical practice guidelines: Management of chronic hepatitis B virus infection. J Hepatol 2012; 57: 167-185.

18. Goertz RS, Sturm J, Zopf S, Wildner D, Neurath MF, Strobel D. Outcome analysis of liver stiffness by ARFI (acoustic radiation force impulse) elastometry in patients with chronic viral hepatitis B and C. Clin Radiol 2014; 65: 275-279.

19. Yoon KT, Lim SM, Park JY, et al. Liver stiffness measurement using acoustic radiation force impulse (ARFI) elastography and effect of necroinflammation. Dig Dis Sci 2012; 57: 1682-1691.
20. Bota S, Sporea I, Peck-Radosavljevic M, et al. The influence of aminotransferase levels on liver stiffness assessed by Acoustic Radiation Force Impulse Elastography: a retrospective multicentre study. Dig Liver Dis 2013; 45: 762768.

21. Coco B, Oliveri F, Maina AM, et al. Transient elastography: a new surrogate marker of liver fibrosis influenced by major changes of transaminases. J Viral Hepat 2007; 14: 360-369.

22. Chan HL, Wong GL, Choi PC, et al. Alanine aminotransferase-based algorithms of liver stiffness measurement by transient elastography (Fibroscan) for liver fibrosis in chronic hepatitis B. J Viral Hepat 2009; 16: 36-44. 